Synthesis and Structure of $\left(\square^{5} \text {-cyclohexadienyl)Mn(CO }\right)_{3}$ and Cationic $\left(\square^{6}\right.$-arene $) M n(C O)_{3}$ Complexes Comprising a Double Bond Conjugated to the $\square$ System.

\begin{abstract}
D. Cetiner ${ }^{\mathrm{a}}$, JP Tranchier ${ }^{\mathrm{a}}$, F. Rose-Munch ${ }^{{ }^{\mathrm{a}} \mathrm{a}}$, E. Rose ${ }^{\mathrm{*}_{\mathrm{a}}}$, P. Herson ${ }^{\mathrm{b}}$
Université Pierre et Marie Curie - Paris 6, Institut de Chimie Moléculaire (FR 2769), 'ababoratoire de Chimie Organique (UMR CNRS 7611), Case 181, ${ }^{\mathrm{b}}$ Laboratoire de Chimie Inorganique et Matériaux Moléculaires (UMR CNRS 7071), Case 42, 4 place Jussieu, F-75252 Paris Cedex 05, France.
\end{abstract}

Supporting Informations 


\section{Complex 7}

\section{TABLE I CRYSTAL DATA}

Formula

$\mathrm{Fw}=$

Crystal System

a $(\AA)=$

$\mathrm{b}(\AA)=$

c $(\AA)=$

$\square\left(^{\circ}\right)=$

$\square\left(^{\circ}\right)=$

$\left[\left(^{\circ}\right)=\right.$

$\mathrm{V}\left(\AA^{3}\right)=$

$\mathrm{Z}=$

Space group

Crystal shape

Crystal colour

Linear absorption coefficient $\square\left(\mathrm{cm}^{-1}\right)$

Density $\square\left(\mathrm{g} \mathrm{cm}^{3}\right)$

Diffractometer

Radiation

Temperature

$\square$ Limits $\left({ }^{\circ}\right)$

Octants collected

$\mathrm{Nb}$ of data collected

$\mathrm{Nb}$ of unique data collected

$\mathrm{Nb}$ of unique data used for refinement

Merging $\mathrm{R}$

$\mathrm{R}=\square|| \mathrm{Fo}|-| \mathrm{Fc} \| / \square \mid \mathrm{Fol}$

$\mathrm{Rw}^{*}=\left[\square \mathrm{w}(\|\mathrm{Fol}-\mathrm{I} \mathrm{Fc}\|)^{2} / \square \mathrm{wFo}^{2}\right]^{1 / 2}$

Absorption correction

Secondary extinction coefficient

Goodness of fit

$\mathrm{Nb}$ of variables

미in $\left(\mathrm{e} / \AA^{3}\right)$

$\square \square \max \left(\mathrm{e} / \AA^{3}\right)$
$\mathrm{C}_{16} \mathrm{H}_{13} \mathrm{MnO}_{4} \mathrm{~S}$

356.27

Monoclinic

6.7948 (6)

$19.899(3)$

$12.1956(15)$

90

$101.939(10)$

90

$1613.3(3)$

4

$\mathrm{P} 2 / \mathrm{n}$

Parallelepiped

yellow

9.61

1.47

KAPPACCD-Enraf Nonius

$\operatorname{MoK} \square(\square=0.71073 \AA$ )

$250 \mathrm{~K}$

$2-30$

$-9,6 ;-27,27 ;-16,17$

15998

4629

$2775(\mathrm{Fo})^{2}>3 \square(\mathrm{Fo})^{2}$

0.043

0.0404

0.0438

sadabs

54.905

1.069

201

$-0.612$

0.587 
* Weighting scheme of the form $w=w^{\prime}\left[1-((\| F o|-| F c||) / 6 \square(F o))^{2}\right]^{2}$ with $w^{\prime}=1 / \square_{r}$ $\mathrm{A}_{\mathrm{r}} \mathrm{T}_{\mathrm{r}}(\mathrm{X})$ with coefficients $1.63,-0.564,1.46,-0.206$ and 0.268 for a Chebyshev serie for which $\mathrm{X}=\mathrm{Fc} / \mathrm{Fc}(\max )$

Table II : Fractional atomic coordinates for $\mathrm{C}_{16} \mathrm{H}_{13} \mathrm{MnO}_{4} \mathrm{~S}$

\begin{tabular}{|c|c|c|c|c|}
\hline Atom & $\mathrm{x} / \mathrm{a}$ & $y / b$ & $z / c$ & $\mathrm{U}$ (eqv) \\
\hline $\operatorname{Mn}(1)$ & $0.17707(5)$ & $0.624158(16)$ & $0.17062(3)$ & 0.0322 \\
\hline$S(1)$ & $0.28543(16)$ & $0.61145(5)$ & $-0.24579(8)$ & 0.0754 \\
\hline$O(1)$ & $0.4803(4)$ & $0.55518(10)$ & $0.37452(16)$ & 0.0587 \\
\hline$O(14)$ & $0.0302(4)$ & $0.76397(10)$ & $0.1291(2)$ & 0.0676 \\
\hline $\mathrm{O}(15)$ & $-0.1770(3)$ & $0.56044(12)$ & $0.0232(2)$ & 0.0639 \\
\hline$O(16)$ & $-0.0184(4)$ & $0.58715(13)$ & $0.3589(2)$ & 0.0737 \\
\hline$C(1)$ & $0.4010(3)$ & $0.65358(11)$ & $0.06271(19)$ & 0.0332 \\
\hline$C(2)$ & $0.3743(3)$ & $0.58336(11)$ & $0.07075(19)$ & 0.0343 \\
\hline$C(3)$ & $0.4081(4)$ & $0.54977(12)$ & $0.1768(2)$ & 0.0393 \\
\hline$C(4)$ & $0.4649(4)$ & $0.58947(13)$ & $0.2757(2)$ & 0.0423 \\
\hline$C(5)$ & $0.4749(4)$ & $0.65984(13)$ & $0.2654(2)$ & 0.0425 \\
\hline$C(6)$ & $0.5350(4)$ & $0.68898(13)$ & $0.1620(2)$ & 0.0406 \\
\hline$C(7)$ & $0.5233(7)$ & $0.5943(2)$ & $0.4766(3)$ & 0.0780 \\
\hline$C(8)$ & $0.3549(4)$ & $0.68749(12)$ & $-0.0480(2)$ & 0.0390 \\
\hline$C(9)$ & $0.4446(6)$ & $0.74514(17)$ & $-0.0677(3)$ & 0.0646 \\
\hline$C(10)$ & $0.2075(4)$ & $0.65518(12)$ & $-0.1414(2)$ & 0.0413 \\
\hline$C(11)$ & $-0.0074(4)$ & $0.65539(14)$ & $-0.1584(2)$ & 0.0448 \\
\hline$C(12)$ & $-0.0926(6)$ & $0.6189(2)$ & $-0.2593(3)$ & 0.0692 \\
\hline$C(13)$ & $0.0433(7)$ & $0.59233(19)$ & $-0.3115(3)$ & 0.0774 \\
\hline$C(14)$ & $0.0837(4)$ & $0.70959(12)$ & $0.1483(2)$ & 0.0419 \\
\hline$C(15)$ & $-0.0375(4)$ & $0.58527(12)$ & $0.0783(2)$ & 0.0419 \\
\hline$C(16)$ & $0.0578(5)$ & $0.60235(14)$ & $0.2865(2)$ & 0.0482 \\
\hline
\end{tabular}


Table III : Interatomic distances ( $\AA$ ) for $\mathrm{C}_{16} \mathrm{H}_{13} \mathrm{MnO}_{4} \mathrm{~S}$

$\begin{array}{llll}M n(1)-C(1) & 2.285(2) & M n(1)-C(2) & 2.148(2) \\ M n(1)-C(3) & 2.148(2) & M n(1)-C(4) & 2.215(3) \\ M n(1)-C(5) & 2.229(2) & M n(1)-C(14) & 1.815(3) \\ M n(1)-C(15) & 1.820(3) & M n(1)-C(16) & 1.821(3) \\ S(1)-C(10) & 1.714(3) & S(1)-C(13) & 1.717(4) \\ O(1)-C(4) & 1.370(3) & O(1)-C(7) & 1.446(4) \\ O(14)-C(14) & 1.150(3) & O(15)-C(15) & 1.154(3) \\ O(16)-C(16) & 1.153(3) & C(1)-C(2) & 1.415(3) \\ C(1)-C(6) & 1.528(3) & C(1)-C(8) & 1.484(3) \\ C(2)-C(3) & 1.431(3) & C(3)-C(4) & 1.427(4) \\ C(4)-C(5) & 1.409(3) & C(5)-C(6) & 1.518(4) \\ C(8)-C(9) & 1.344(4) & C(8)-C(10) & 1.497(4) \\ C(10)-C(11) & 1.431(4) & C(11)-C(12) & 1.442(4) \\ C(12)-C(13) & 1.335(6) & & \end{array}$

Table IV :Bond angles $\left({ }^{\circ}\right)$ for $\mathrm{C}_{16} \mathrm{H}_{13} \mathrm{MnO}_{4} \mathrm{~S}$

\begin{tabular}{|c|c|c|c|c|c|c|c|}
\hline$C(1)$ & $-\operatorname{Mn}(1)$ & $-C(2)$ & $37.07(8)$ & $C(1)$ & $-\operatorname{Mn}(1)$ & $-C(3)$ & $68.13(9)$ \\
\hline$C(2)$ & $-\operatorname{Mn}(1)$ & $-C(3)$ & $38.92(9)$ & $C(1)$ & $-\operatorname{Mn}(1)$ & $-C(4)$ & $78.44(9)$ \\
\hline$C(2)$ & - Mn (1) & $-C(4)$ & $68.32(9)$ & $C(3)$ & $-\operatorname{Mn}(1)$ & $-C(4)$ & $38.14(9)$ \\
\hline$C(1)$ & $-\operatorname{Mn}(1)$ & $-C(5)$ & $64.88(9)$ & $C(2)$ & $-\operatorname{Mn}(1)$ & $-C(5)$ & $78.95(9)$ \\
\hline$C(3)$ & - Mn (1) & $-C(5)$ & $67.83(10)$ & $C(4)$ & $-\operatorname{Mn}(1)$ & $-C(5)$ & $36.97(9)$ \\
\hline$C(1)$ & $-M n(1)$ & $-C(14)$ & $85.83(10)$ & $C(2)$ & $-M n(1)$ & $-C(14)$ & $120.56(10)$ \\
\hline$C(3)$ & $-\operatorname{Mn}(1)$ & - C (14) & $151.75(11)$ & $C(4)$ & $-\operatorname{Mn}(1)$ & $-C(14)$ & $128.18(11)$ \\
\hline$C(5)$ & - Mn(1) & - C (14) & $91.79(10)$ & $C(1)$ & $-\operatorname{Mn}(1)$ & $-C(15)$ & $107.24(10)$ \\
\hline$C(2)$ & $-\operatorname{Mn}(1)$ & - C(15) & $90.14(10)$ & $C(3)$ & $-\operatorname{Mn}(1)$ & - C (15) & $102.40(10)$ \\
\hline$C(4)$ & - Mn (1) & - C(15) & $136.36(11)$ & $C(5)$ & $-\operatorname{Mn}(1)$ & $-C(15)$ & $168.93(11)$ \\
\hline$C(1)$ & $-\operatorname{Mn}(1)$ & $-C(16)$ & $164.71(11)$ & $C(2)$ & $-\operatorname{Mn}(1)$ & - C (16) & $141.26(11)$ \\
\hline$C(3)$ & - Mn (1) & $-C(16)$ & $104.37(11)$ & $C(4)$ & $-\operatorname{Mn}(1)$ & $-C(16)$ & $87.60(12)$ \\
\hline$C(5)$ & $-\operatorname{Mn}(1)$ & $-C(16)$ & $100.12(12)$ & $C(14)$ & $-\operatorname{Mn}(1)$ & - C (15) & $95.45(12)$ \\
\hline$C(14)$ & $-\operatorname{Mn}(1)$ & $-C(16)$ & $98.16(12)$ & $C(15)$ & $-\operatorname{Mn}(1)$ & $-C(16)$ & $87.16(12)$ \\
\hline$C(10)$ & $-S(1)$ & $-C(13)$ & $92.62(18)$ & $C(4)$ & $-O(1)$ & $-C(7)$ & $117.0(2)$ \\
\hline $\operatorname{Mn}(1)$ & $-C(1)$ & $-C(2)$ & $66.22(13)$ & $\mathrm{Mn}(1)$ & $-C(1)$ & $-C(6)$ & $91.16(14)$ \\
\hline$C(2)$ & - C(1) & $-C(6)$ & $117.7(2)$ & Mn (1) & $-C(1)$ & $-C(8)$ & $126.87(16)$ \\
\hline$C(2)$ & $-C(1)$ & $-C(8)$ & $120.4(2)$ & $C(6)$ & $-C(1)$ & $-C(8)$ & $119.4(2)$ \\
\hline $\operatorname{Mn}(1)$ & $-C(2)$ & $-C(1)$ & $76.71(14)$ & $\mathrm{Mn}(1)$ & $-C(2)$ & $-C(3)$ & $70.52(14)$ \\
\hline$C(1)$ & $-C(2)$ & $-C(3)$ & $121.7(2)$ & Mn (1) & $-C(3)$ & $-C(2)$ & $70.55(13)$ \\
\hline $\mathrm{Mn}(1)$ & - C(3) & $-C(4)$ & $73.49(14)$ & $C(2)$ & $-C(3)$ & $-C(4)$ & $118.1(2)$ \\
\hline $\operatorname{Mn}(1)$ & $-C(4)$ & $-O(1)$ & $124.2(2)$ & $\mathrm{Mn}(1)$ & $-C(4)$ & $-C(3)$ & $68.37(13)$ \\
\hline$O(1)$ & $-C(4)$ & $-C(3)$ & $115.2(2)$ & Mn (1) & $-C(4)$ & $-C(5)$ & $72.03(15)$ \\
\hline$O(1)$ & $-C(4)$ & $-C(5)$ & $125.3(2)$ & $C(3)$ & $-C(4)$ & $-C(5)$ & $118.9(2)$ \\
\hline $\mathrm{Mn}(1)$ & $-C(5)$ & $-C(4)$ & $71.00(15)$ & Mn (1) & $-C(5)$ & $-C(6)$ & $93.60(15)$ \\
\hline$C(4)$ & $-C(5)$ & $-C(6)$ & $118.7(2)$ & $C(1)$ & $-C(6)$ & $-C(5)$ & $105.26(19)$ \\
\hline$C(1)$ & $-C(8)$ & $-C(9)$ & $122.2(2)$ & $C(1)$ & $-C(8)$ & $-C(10)$ & $118.6(2)$ \\
\hline$C(9)$ & $-C(8)$ & $-C(10)$ & $119.2(2)$ & $S(1)$ & $-C(10)$ & $-C(8)$ & $121.5(2)$ \\
\hline$S(1)$ & $-C(10)$ & $-C(11)$ & $110.9(2)$ & $C(8)$ & $-C(10)$ & $-C(11)$ & $127.7(2)$ \\
\hline$C(10)$ & $-C(11)$ & $-C(12)$ & $109.9(3)$ & $C(11)$ & $-C(12)$ & $-C(13)$ & $114.3(3)$ \\
\hline$S(1)$ & $-C(13)$ & $-C(12)$ & $112.3(3)$ & $\operatorname{Mn}(1)$ & $-C(14)$ & $-O(14)$ & $176.1(2)$ \\
\hline $\operatorname{Mn}(1)$ & $-C(15)$ & $-O(15)$ & $177.4(2)$ & $\mathrm{Mn}(1)$ & $-C(16)$ & $-O(16)$ & $178.5(2)$ \\
\hline
\end{tabular}


Table S1 : Anisotropic thermal parameters for $\mathrm{C}_{16} \mathrm{H}_{13} \mathrm{MnO}_{4} \mathrm{~S}$

$\begin{array}{llllrrr}\text { Atom } & \mathrm{u}(11) & \mathrm{u}(22) & \mathrm{u}(33) & \mathrm{u}(23) & \mathrm{u}(13) & \mathrm{u}(12) \\ \mathrm{Mn}(1) & 0.03653(18) & 0.02776(16) & 0.03206(18) & -0.00218(14) & 0.00669(12) & -0.00361(15) \\ \mathrm{S}(1) & 0.0827(6) & 0.0837(7) & 0.0578(5) & -0.0174(4) & 0.0098(4) & 0.0163(5) \\ O(1) & 0.0852(15) & 0.0502(11) & 0.0330(9) & 0.0079(8) & -0.0054(9) & -0.0050(11) \\ O(14) & 0.0703(15) & 0.0375(10) & 0.0982(18) & 0.0060(11) & 0.0246(13) & 0.0115(10) \\ O(15) & 0.0454(11) & 0.0667(13) & 0.0772(15) & -0.0246(11) & 0.0074(10) & -0.0177(10) \\ O(16) & 0.1002(19) & 0.0695(15) & 0.0637(15) & 0.0043(11) & 0.0455(14) & -0.0088(14) \\ C(1) & 0.0296(10) & 0.0336(10) & 0.0352(11) & 0.0008(8) & 0.0037(9) & -0.0007(8) \\ C(2) & 0.0349(11) & 0.0321(10) & 0.0348(11) & -0.0017(8) & 0.0045(9) & 0.0022(9) \\ C(3) & 0.0449(13) & 0.0323(11) & 0.0377(12) & 0.0028(9) & 0.0016(10) & 0.0038(9) \\ C(4) & 0.0475(14) & 0.0437(13) & 0.0306(12) & 0.0055(10) & -0.0036(10) & 0.0001(11) \\ C(5) & 0.0454(14) & 0.0424(13) & 0.0353(12) & -0.0030(10) & -0.0022(10) & -0.0066(10) \\ C(6) & 0.0364(12) & 0.0419(12) & 0.0405(13) & 0.0006(10) & 0.0008(10) & -0.0075(10) \\ C(7) & 0.120(3) & 0.073(2) & 0.0310(15) & 0.0034(14) & -0.0074(17) & -0.018(2) \\ C(8) & 0.0393(12) & 0.0404(12) & 0.0374(12) & 0.0048(9) & 0.0082(10) & -0.0006(10) \\ C(9) & 0.082(2) & 0.0603(18) & 0.0482(17) & 0.0151(14) & 0.0063(15) & -0.0245(16) \\ C(10) & 0.0526(14) & 0.0382(12) & 0.0316(12) & 0.0070(9) & 0.0053(10) & 0.0023(10) \\ C(11) & 0.0393(13) & 0.0587(15) & 0.0300(12) & 0.0059(10) & -0.0075(10) & -0.0032(11) \\ C(12) & 0.069(2) & 0.076(2) & 0.0511(17) & 0.0102(17) & -0.0136(15) & -0.0155(18) \\ C(13) & 0.106(3) & 0.061(2) & 0.0517(19) & -0.0090(15) & -0.015(2) & -0.001(2) \\ C(14) & 0.0401(13) & 0.0361(12) & 0.0510(15) & -0.0035(10) & 0.0129(11) & -0.0037(10) \\ C(15) & 0.0407(13) & 0.0379(12) & 0.0483(14) & -0.0078(10) & 0.0117(11) & -0.0055(10) \\ C(16) & 0.0622(17) & 0.0393(12) & 0.0451(15) & -0.0035(11) & 0.0158(13) & -0.0040(12)\end{array}$

$\mathrm{C}_{16} \mathrm{H}_{13} \mathrm{MnO}_{4} \mathrm{~S}$

Table S2 : Hydrogen atoms fractional atomic coordinates for

Atom
H (21)
$H(31)$
$H(51)$
$H(61)$
$H(62)$
$H(71)$
$H(72)$
$H(73)$
$H(91)$
$H(92)$
$H(111)$
$H(121)$
$H(131)$

$\mathbf{x} / \mathbf{a}$

0.3308

0.3924

0.4430

0.5111

0.6801

0.5308

0.6548

0.4141

0.5463

0.4097

$-0.0866$

$-0.2409$

0.0082

$$
\mathrm{y} / \mathrm{b}
$$

0.5565

0.4999

0.6900

0.7386

0.6797

0.5638

0.6180

0.6281

0.7666

0.7666

0.6775

0.6140

0.5644 $z / c \quad U($ iso)

$\begin{array}{rr}0.0006 & 0.074(3) \\ 0.1814 & 0.074(3) \\ 0.3250 & 0.074(3) \\ 0.1578 & 0.074(3) \\ 0.1633 & 0.074(3) \\ 0.5426 & 0.074(3) \\ 0.4824 & 0.074(3) \\ 0.4755 & 0.074(3) \\ -0.0067 & 0.074(3) \\ -0.1434 & 0.074(3) \\ -0.1079 & 0.074(3) \\ -0.2877 & 0.074(3) \\ -0.3810 & 0.074(3)\end{array}$




\section{Complex 11}

TABLE I CRYSTAL DATA

Formula

$\mathrm{Fw}=$

Crystal System

C16H12BF4MnO4S

442.07

Monoclinic

a $(\AA)=$

$\mathrm{b}(\AA)=$

16.8079 (7)

c $(\AA)=$

$7.1173(3)$

$\square\left({ }^{\circ}\right)=$

$\square\left({ }^{\circ}\right)=$

$\left[\left(^{\circ}\right)=\right.$

29.7749 (17)

90

90.603 (4)

90

$\mathrm{V}\left(\AA^{3}\right)=$

$\mathrm{Z}=$

$3558.1(3)$

Space group

8

Crystal shape

$\mathrm{P} 2{ }_{1} / \mathrm{c}$

Parallelepiped

Crystal colour

Linear absorption coefficient $\square\left(\mathrm{cm}^{-1}\right)$

yellow

Density $\square\left(\mathrm{g} \mathrm{cm}^{3}\right)$

9.19

1.65

Diffractometer

Radiation

Temperature

$\square$ Limits $\left({ }^{\circ}\right)$

KAPPACCD-Enraf Nonius

$\operatorname{MoK} \square(\square=0.71073 \AA$ )

$150 \mathrm{~K}$

2-30

Octants collected

$-22,23 ;-9,10 ;-41,38$

$\mathrm{Nb}$ of data collected

52256

$\mathrm{Nb}$ of unique data collected

10041

$\mathrm{Nb}$ of unique data used for refinement

$6347(\mathrm{Fo})^{2}>3 \square(\mathrm{Fo})^{2}$

Merging R

0.0396

$\mathrm{R}=\square|| \mathrm{Fo}|-| \mathrm{Fc} \| / \mathrm{I} \mid \mathrm{Fol}$

0.0344

$\mathrm{Rw}^{*}=\left[\square \mathrm{w}(\|\mathrm{Fol}-\mid \mathrm{Fc}\|)^{2} / \square \mathrm{wFo}^{2}\right]^{1 / 2}$

0.0382

Absorption correction

sadabs

Secondary extinction coefficient

18.88

Goodness of fit

1.108

$\mathrm{Nb}$ of variables

507

$\square \square \min \left(\mathrm{e} / \AA^{3}\right)$

$-0.44$

$\square \square \max \left(\mathrm{e} / \AA^{3}\right)$

1.40

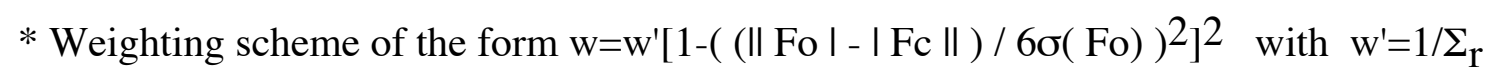
$\mathrm{A}_{\mathrm{r}} \mathrm{T}_{\mathrm{r}}(\mathrm{X})$ with coefficients $0.981,0.101$ and 0.612 for a Chebyshev serie for which $\mathrm{X}=\mathrm{Fc} / \mathrm{Fc}(\max )$ 
Table II : Fractional atomic coordinates for $\mathrm{C}_{16} \mathrm{H}_{12} \mathrm{BF}_{4} \mathrm{MnO}_{4} \mathrm{~S}$

\begin{tabular}{|c|c|c|c|c|c|}
\hline Atom & $\mathrm{x} / \mathrm{a}$ & $\mathrm{y} / \mathrm{b}$ & $z / c$ & $\mathrm{U}$ (eqv) & Occ \\
\hline $\operatorname{Mn}(1)$ & $0.328625(18)$ & $0.82939(4)$ & $0.236555(10)$ & 0.0163 & \\
\hline $\operatorname{Mn}(2)$ & $0.257898(18)$ & $0.87324(4)$ & $0.489286(11)$ & 0.0179 & \\
\hline$S(1)$ & $-0.01853(3)$ & $0.70515(8)$ & $0.23050(2)$ & 0.0265 & \\
\hline$S(2)$ & $-0.07350(3)$ & $0.72794(8)$ & $0.433624(18)$ & 0.0220 & \\
\hline$O(7)$ & $0.45654(9)$ & $0.5845(3)$ & $0.17624(6)$ & 0.0299 & \\
\hline$O(14)$ & $0.21725(10)$ & $1.1484(2)$ & $0.24804(7)$ & 0.0333 & \\
\hline$O(15)$ & $0.38858(11)$ & $0.8374(3)$ & $0.33046(5)$ & 0.0347 & \\
\hline$O(16)$ & $0.45679(11)$ & $1.0926(3)$ & $0.21087(7)$ & 0.0414 & \\
\hline$O(27)$ & $0.41083(18)$ & $0.6119(5)$ & $0.45977(14)$ & 0.0258 & 0.5000 \\
\hline$O(34)$ & $0.16751(11)$ & $1.2157(2)$ & $0.46481(6)$ & 0.0323 & \\
\hline$O(35)$ & $0.21804(12)$ & $0.9061(3)$ & $0.58564(6)$ & 0.0398 & \\
\hline$O(36)$ & $0.40938(12)$ & $1.0853(3)$ & $0.49770(8)$ & 0.0492 & \\
\hline$O(271)$ & $0.4084(2)$ & $0.6589(6)$ & $0.43557(15)$ & 0.0291 & 0.5000 \\
\hline$C(1)$ & $0.22547(12)$ & $0.6413(3)$ & $0.22724(7)$ & 0.0181 & \\
\hline$C(2)$ & $0.24566(11)$ & $0.7286(3)$ & $0.18557(7)$ & 0.0174 & \\
\hline$C(3)$ & $0.32274(12)$ & $0.7158(3)$ & $0.16794(7)$ & 0.0194 & \\
\hline$C(4)$ & $0.38186(12)$ & $0.6069(3)$ & $0.19053(7)$ & 0.0215 & \\
\hline$C(5)$ & $0.36396(13)$ & $0.5305(3)$ & $0.23290(8)$ & 0.0233 & \\
\hline$C(6)$ & $0.28603(13)$ & $0.5447(3)$ & $0.25053(7)$ & 0.0211 & \\
\hline$C(7)$ & $0.47866(14)$ & $0.6715(4)$ & $0.13385(9)$ & 0.0345 & \\
\hline$C(8)$ & $0.14384(12)$ & $0.6581(3)$ & $0.24669(7)$ & 0.0212 & \\
\hline$C(9)$ & $0.13491(15)$ & $0.6904(4)$ & $0.29082(8)$ & 0.0335 & \\
\hline$C(10)$ & $0.07605(12)$ & $0.6339(3)$ & $0.21559(7)$ & 0.0205 & \\
\hline$C(11)$ & $0.07512(12)$ & $0.5485(3)$ & $0.17331(8)$ & 0.0246 & \\
\hline$C(12)$ & $-0.00339(14)$ & $0.5453(4)$ & $0.15428(9)$ & 0.0327 & \\
\hline$C(13)$ & $-0.05909(14)$ & $0.6241(4)$ & $0.18116(9)$ & 0.0318 & \\
\hline$C(14)$ & $0.26154(13)$ & $1.0290(3)$ & $0.24429(7)$ & 0.0230 & \\
\hline$C(15)$ & $0.36429(13)$ & $0.8395(3)$ & $0.29477(7)$ & 0.0245 & \\
\hline$C(16)$ & $0.40736(13)$ & $0.9909(3)$ & $0.22058(8)$ & 0.0260 & \\
\hline$C(21)$ & $0.16392(12)$ & $0.6810(3)$ & $0.46641(7)$ & 0.0202 & \\
\hline$C(22)$ & $0.20178(12)$ & $0.7629(3)$ & $0.42873(8)$ & 0.0247 & \\
\hline$C(23)$ & $0.28485(13)$ & $0.7521(4)$ & $0.42312(9)$ & 0.0328 & \\
\hline$C(24)$ & $0.33195(14)$ & $0.6481(4)$ & $0.45411(12)$ & 0.0409 & \\
\hline$C(25)$ & $0.29689(14)$ & $0.5766(3)$ & $0.49285(12)$ & 0.0401 & \\
\hline$C(26)$ & $0.21344(14)$ & $0.5901(3)$ & $0.49834(9)$ & 0.0290 & \\
\hline$C(27)$ & $0.4535(3)$ & $0.6744(9)$ & $0.42059(19)$ & 0.0385 & 0.5000 \\
\hline$C(28)$ & $0.07616(11)$ & $0.6975(3)$ & $0.47369(7)$ & 0.0187 & \\
\hline$C(29)$ & $0.04703(13)$ & $0.7438(4)$ & $0.51389(8)$ & 0.0284 & \\
\hline$C(30)$ & $0.02486(11)$ & $0.6550(3)$ & $0.43464(7)$ & 0.0181 & \\
\hline$C(31)$ & $0.04181(12)$ & $0.5505(3)$ & $0.39698(8)$ & 0.0228 & \\
\hline$C(32)$ & $-0.02513(14)$ & $0.5307(3)$ & $0.36761(8)$ & 0.0270 & \\
\hline$C(33)$ & $-0.09168(13)$ & $0.6188(3)$ & $0.38302(8)$ & 0.0254 & \\
\hline$C(34)$ & $0.20407(13)$ & $1.0871(3)$ & $0.47522(7)$ & 0.0211 & \\
\hline$C(35)$ & $0.23544(14)$ & $0.9009(3)$ & $0.54892(8)$ & 0.0270 & \\
\hline$C(36)$ & $0.35132(14)$ & $1.0055(4)$ & $0.49516(9)$ & 0.0311 & \\
\hline$C(271)$ & $0.4632(3)$ & $0.5578(8)$ & $0.4641(2)$ & 0.0371 & 0.5000 \\
\hline$B(1)$ & $0.71180(16)$ & $0.7568(5)$ & $0.13705(9)$ & 0.0325 & \\
\hline$B(2)$ & $0.31882(15)$ & $0.1847(5)$ & $0.10409(10)$ & 0.0319 & \\
\hline$F(11)$ & $0.76333(12)$ & $0.9087(3)$ & $0.13904(7)$ & 0.0556 & \\
\hline$F(12)$ & $0.65934(10)$ & $0.7654(3)$ & $0.17311(5)$ & 0.0439 & \\
\hline$F(13)$ & $0.66974(10)$ & $0.7538(3)$ & $0.09671(5)$ & 0.0492 & \\
\hline$F(14)$ & $0.75560(11)$ & $0.5896(3)$ & $0.14137(6)$ & 0.0477 & \\
\hline$F(21)$ & $0.36589(11)$ & $0.3442(3)$ & $0.10253(7)$ & 0.0579 & \\
\hline$F(22)$ & $0.28299(11)$ & $0.1686(4)$ & $0.14482(6)$ & 0.0695 & \\
\hline$F(23)$ & $0.26037(10)$ & $0.2015(3)$ & $0.06959(6)$ & 0.0432 & \\
\hline$F(24)$ & $0.36531(11)$ & $0.0269(3)$ & $0.09432(7)$ & 0.0557 & \\
\hline
\end{tabular}


Table III : Interatomic distances ( $\AA$ ) for $\mathrm{C}_{16} \mathrm{H}_{12} \mathrm{BF}_{4} \mathrm{MnO}_{4} \mathrm{~S}$

$\begin{array}{llll}M n(1)-C(1) & 2.206(2) & M n(1)-C(2) & 2.1713(19) \\ M n(1)-C(3) & 2.196(2) & M n(1)-C(4) & 2.282(2) \\ M n(1)-C(5) & 2.212(2) & M n(1)-C(6) & 2.190(2) \\ M n(1)-C(14) & 1.830(2) & M n(1)-C(15) & 1.828(2) \\ M n(1)-C(16) & 1.820(2) & M n(2)-C(21) & 2.193(2) \\ M n(2)-C(22) & 2.171(2) & M n(2)-C(23) & 2.200(2) \\ M n(2)-C(24) & 2.289(2) & M n(2)-C(25) & 2.213(2) \\ M n(2)-C(26) & 2.167(2) & M n(2)-C(34) & 1.817(2) \\ M n(2)-C(35) & 1.828(2) & M n(2)-C(36) & 1.838(2) \\ S(1)-C(10) & 1.731(2) & \mathrm{S}(1)-C(13) & 1.712(3) \\ S(2)-C(30) & 1.733(2) & \mathrm{S}(2)-C(33) & 1.718(2) \\ O(7)-C(4) & 1.339(3) & O(7)-C(7) & 1.456(3) \\ O(14)-C(14) & 1.136(3) & O(15)-C(15) & 1.133(3) \\ O(16)-C(16) & 1.141(3) & O(27)-C(24) & 1.359(4) \\ O(27)-C(27) & 1.445(6) & O(34)-C(34) & 1.143(3) \\ O(35)-C(35) & 1.134(3) & O(36)-C(36) & 1.131(3) \\ O(271)-C(24) & 1.405(4) & O(271)-C(271) & 1.439(6) \\ C(1)-C(2) & 1.431(3) & C(1)-C(6) & 1.405(3) \\ C(1)-C(8) & 1.499(3) & C(2)-C(3) & 1.406(3) \\ C(3)-C(4) & 1.423(3) & C(4)-C(5) & 1.408(3) \\ C(5)-C(6) & 1.420(3) & C(8)-C(9) & 1.342(3) \\ C(8)-C(10) & 1.470(3) & C(10)-C(11) & 1.396(3) \\ C(11)-C(12) & 1.431(3) & C(12)-C(13) & 1.359(4) \\ C(21)-C(22) & 1.420(3) & C(21)-C(26) & 1.413(3) \\ C(21)-C(28) & 1.498(3) & C(22)-C(23) & 1.410(3) \\ C(23)-C(24) & 1.417(4) & C(24)-C(25) & 1.396(5) \\ C(25)-C(26) & 1.417(3) & C(27)-C(271) & 1.545(8) \\ C(28)-C(29) & 1.338(3) & C(28)-C(30) & 1.471(3) \\ C(30)-C(31) & 1.377(3) & C(31)-C(32) & 1.424(3) \\ C(32)-C(33) & 1.366(3) & B(1)-F(11) & 1.386(3) \\ B(1)-F(12) & 1.397(3) & B(1)-F(13) & 1.386(3) \\ B(1)-F(14) & 1.405(4) & B(2)-F(21) & 1.385(3) \\ B(2)-F(22) & 1.363(3) & B(2)-F(23) & 1.418(3) \\ B(2)-F(24) & 1.400(4) & & \end{array}$


Table IV : Bond angles $\left({ }^{\circ}\right.$ ) for $\mathrm{C}_{16} \mathrm{H}_{12} \mathrm{BF}_{4} \mathrm{MnO}_{4} \mathrm{~S}$

\begin{tabular}{|c|c|c|c|c|c|c|c|}
\hline$C(1)$ & $-\operatorname{Mn}(1)$ & $-C(2)$ & $38.15(7)$ & $C(1)$ & - $M n(1)$ & $-c(3)$ & $68.43(7)$ \\
\hline$C(2)$ & $-\operatorname{Mn}(1)$ & $-C(3)$ & $37.54(7)$ & $C(1)$ & $-\operatorname{Mn}(1)$ & $-C(4)$ & $79.40(8)$ \\
\hline$C(2)$ & $-\operatorname{Mn}(1)$ & $-C(4)$ & $66.72(7)$ & $C(3)$ & $-\operatorname{Mn}(1)$ & $-C(4)$ & $37.00(8)$ \\
\hline$C(1)$ & $-\operatorname{Mn}(1)$ & $-C(5)$ & $67.74(8)$ & $C(2)$ & $-\operatorname{Mn}(1)$ & $-C(5)$ & $79.56(8)$ \\
\hline$C(3)$ & $-\operatorname{Mn}(1)$ & $-C(5)$ & $67.04(8)$ & $C(4)$ & $-\operatorname{Mn}(1)$ & $-C(5)$ & $36.48(8)$ \\
\hline$C(1)$ & - Mn (1) & - C(6) & $37.28(8)$ & $C(2)$ & - Mn (1) & $-C(6)$ & $67.55(8)$ \\
\hline$C(3)$ & - Mn (1) & $-C(6)$ & $79.88(8)$ & $C(4)$ & $-M n(1)$ & $-C(6)$ & $66.60(8)$ \\
\hline$C(5)$ & - Mn (1) & $-C(6)$ & $37.62(8)$ & $C(1)$ & $-M n(1)$ & $-C(14)$ & $90.14(9)$ \\
\hline$C(2)$ & $-\operatorname{Mn}(1)$ & $-C(14)$ & $87.26(9)$ & $C(3)$ & $-\operatorname{Mn}(1)$ & $-C(14)$ & $112.38(9)$ \\
\hline$C(4)$ & $-\operatorname{Mn}(1)$ & $-C(14)$ & $149.33(9)$ & $C(5)$ & $-M n(1)$ & $-C(14)$ & $156.80(9)$ \\
\hline$C(1)$ & - Mn (1) & $-C(15)$ & $113.10(9)$ & $C(2)$ & - Mn (1) & $-C(15)$ & $151.22(9)$ \\
\hline$C(3)$ & $-\operatorname{Mn}(1)$ & $-C(15)$ & $154.95(9)$ & $C(4)$ & $-M n(1)$ & $-C(15)$ & $117.97(9)$ \\
\hline$C(5)$ & - Mn (1) & $-C(15)$ & $89.94(9)$ & $C(1)$ & $-M n(1)$ & $-C(16)$ & $157.44(9)$ \\
\hline$C(2)$ & $-\operatorname{Mn}(1)$ & $-C(16)$ & $119.32(9)$ & $C(3)$ & $-\operatorname{Mn}(1)$ & $-C(16)$ & $90.90(9)$ \\
\hline$C(4)$ & $-\operatorname{Mn}(1)$ & $-C(16)$ & $89.49(9)$ & $C(5)$ & $-M n(1)$ & $-C(16)$ & $113.49(10)$ \\
\hline$C(6)$ & $-\operatorname{Mn}(1)$ & $-C(14)$ & $119.43(9)$ & $C(6)$ & $-M n(1)$ & $-C(15)$ & $87.78(9)$ \\
\hline$C(14)$ & - Mn (1) & $-C(15)$ & $92.67(10)$ & $C(6)$ & $-M n(1)$ & $-C(16)$ & $150.93(10)$ \\
\hline$C(14)$ & $-\operatorname{Mn}(1)$ & $-C(16)$ & $89.60(10)$ & $C(15)$ & $-\operatorname{Mn}(1)$ & $-C(16)$ & $89.45(10)$ \\
\hline$C(21)$ & $-\operatorname{Mn}(2)$ & $-C(22)$ & $37.97(8)$ & $C(21)$ & $-\operatorname{Mn}(2)$ & - C(23) & $68.43(8)$ \\
\hline$C(22)$ & $-\operatorname{Mn}(2)$ & $-C(23)$ & $37.63(8)$ & $C(21)$ & $-\operatorname{Mn}(2)$ & $-C(24)$ & $79.33(8)$ \\
\hline$C(22)$ & $-\operatorname{Mn}(2)$ & $-C(24)$ & $66.50(9)$ & $C(23)$ & $-\operatorname{Mn}(2)$ & $-C(24)$ & $36.74(11)$ \\
\hline$C(21)$ & $-\operatorname{Mn}(2)$ & $-C(25)$ & $68.38(8)$ & $C(22)$ & $-\operatorname{Mn}(2)$ & $-C(25)$ & $79.66(10)$ \\
\hline$C(23)$ & $-\operatorname{Mn}(2)$ & $-C(25)$ & $66.76(12)$ & $C(24)$ & $-\operatorname{Mn}(2)$ & $-C(25)$ & $36.08(12)$ \\
\hline$C(21)$ & $-\operatorname{Mn}(2)$ & $-C(26)$ & $37.82(8)$ & $C(22)$ & $-\operatorname{Mn}(2)$ & $-C(26)$ & $67.63(10)$ \\
\hline$C(23)$ & $-\operatorname{Mn}(2)$ & $-C(26)$ & $79.70(10)$ & $C(24)$ & $-\operatorname{Mn}(2)$ & $-C(26)$ & $66.15(10)$ \\
\hline$C(25)$ & $-\operatorname{Mn}(2)$ & $-C(26)$ & $37.73(9)$ & $C(21)$ & $-\operatorname{Mn}(2)$ & $-c(34)$ & $0(9)$ \\
\hline$C(22)$ & $-\operatorname{Mn}(2)$ & $-c(34)$ & $84.31(9)$ & $C(23)$ & $-\operatorname{Mn}(2)$ & $-C(34)$ & $103.23(10)$ \\
\hline$C(24)$ & $-\operatorname{Mn}(2)$ & $-c(34)$ & $138.84(11)$ & $C(25)$ & $-\operatorname{Mn}(2)$ & $-c(34)$ & $163.06(10)$ \\
\hline$C(21)$ & $-\operatorname{Mn}(2)$ & $-C(35)$ & $102.29(9)$ & $C(22)$ & $-\operatorname{Mn}(2)$ & $-C(35)$ & $138.72(9)$ \\
\hline$C(23)$ & $-\operatorname{Mn}(2)$ & $-c(35)$ & $163.08(11)$ & $C(24)$ & $-\operatorname{Mn}(2)$ & $-c(35)$ & $129.62(12)$ \\
\hline$C(25)$ & $-\operatorname{Mn}(2)$ & $-C(35)$ & $96.90(12)$ & $C(21)$ & $-\operatorname{Mn}(2)$ & $-C(36)$ & $164.01(10)$ \\
\hline$C(22)$ & $-\operatorname{Mn}(2)$ & $-C(36)$ & $128.86(10)$ & $C(23)$ & $-\operatorname{Mn}(2)$ & $-C(36)$ & $95.86(10)$ \\
\hline$C(24)$ & $-\operatorname{Mn}(2)$ & $-C(36)$ & $86.20(10)$ & $C(25)$ & $-\operatorname{Mn}(2)$ & $-C(36)$ & $103.41(10)$ \\
\hline$C(26)$ & $-\operatorname{Mn}(2)$ & $-c(34)$ & $129.49(9)$ & $C(26)$ & $-\operatorname{Mn}(2)$ & $-c(35)$ & $84.52(11)$ \\
\hline$C(34)$ & $-\operatorname{Mn}(2)$ & $-C(35)$ & $91.49(10)$ & $C(26)$ & $-\operatorname{Mn}(2)$ & $-C(36)$ & 139. \\
\hline$C(34)$ & $-M n(2)$ & $-C(36)$ & $90.90(10)$ & $C(35)$ & $-\operatorname{Mn}(2)$ & $-C(36)$ & $92.15(11)$ \\
\hline$C(10)$ & $-s(1)$ & $-c(13)$ & $92.30(11)$ & $C(30)$ & $-s(2)$ & $-c(33)$ & $3(10)$ \\
\hline$C(4)$ & $-O(7)$ & $-C(7)$ & $118.23(18)$ & $C(24)$ & $-O(27)$ & $-C(27)$ & $5(4)$ \\
\hline$C(24)$ & $-O(271)$ & $-C(271)$ & $108.8(4)$ & $\operatorname{Mn}(1)$ & $-C(1)$ & $-C(2)$ & $69.62(11)$ \\
\hline $\operatorname{Mn}(1)$ & $-C(1)$ & $-C(6)$ & $70.78(12)$ & $C(2)$ & $-C(1)$ & $-C(6)$ & $117.55(18)$ \\
\hline $\operatorname{Mn}(1)$ & $-C(1)$ & $-C(8)$ & $128.63(14)$ & $C(2)$ & $-C(1)$ & $-c(8)$ & $121.73(18)$ \\
\hline$C(6)$ & $-C(1)$ & $-C(8)$ & $120.67(18)$ & $\operatorname{Mn}(1)$ & $-C(2)$ & $-C(1)$ & $3(11)$ \\
\hline $\operatorname{Mn}(1)$ & $-C(2)$ & $-C(3)$ & $72.20(11)$ & $C(1)$ & $-C(2)$ & $-c(3)$ & $121.53(18)$ \\
\hline $\operatorname{Mn}(1)$ & $-C(3)$ & $-C(2)$ & $70.26(11)$ & $\operatorname{Mn}(1)$ & $-C(3)$ & $-C(4)$ & $7(12)$ \\
\hline$C(2)$ & $-c(3)$ & $-C(4)$ & $120.05(19)$ & $\operatorname{Mn}(1)$ & $-C(4)$ & $-O(7)$ & $130.37(16)$ \\
\hline $\mathrm{Mn}(1)$ & $-C(4)$ & $-c(3)$ & $68.23(12)$ & $O(7)$ & $-C(4)$ & $-c(3)$ & $124.5(2)$ \\
\hline $\operatorname{Mn}(1)$ & $-C(4)$ & $-C(5)$ & $69.05(12)$ & $O(7)$ & $-C(4)$ & $-C(5)$ & $116.61(19)$ \\
\hline$C(3)$ & $-C(4)$ & $-C(5)$ & $118.59(19)$ & $\operatorname{Mn}(1)$ & $-C(5)$ & $-C(4)$ & $7(13)$ \\
\hline $\operatorname{Mn}(1)$ & $-C(5)$ & $-C(6)$ & $70.37(12)$ & $C(4)$ & $-C(5)$ & $-C(6)$ & $120.63(19)$ \\
\hline Mn (1) & $-C(6)$ & $-C(1)$ & $71.95(12)$ & $\operatorname{Mn}(1)$ & $-C(6)$ & $-C(5)$ & $72.01(12)$ \\
\hline$C(1)$ & $-C(6)$ & $-C(5)$ & $121.29(19)$ & $C(1)$ & $-C(8)$ & $-C(9)$ & $120.2(2)$ \\
\hline$C(1)$ & $-C(8)$ & $-C(10)$ & $117.00(18)$ & $C(9)$ & $-C(8)$ & $-C(10)$ & $122.8(2)$ \\
\hline$S(1)$ & $-c(10)$ & $-C(8)$ & $120.80(16)$ & $s(1)$ & $-C(10)$ & $-c(11)$ & $110.90(16)$ \\
\hline$C(8)$ & $-C(10)$ & $-C(11)$ & $128.24(19)$ & $C(10)$ & $-C(11)$ & $-C(12)$ & $111.4(2)$ \\
\hline$C(11)$ & $-c(12)$ & $-c(13)$ & $113.5(2)$ & $S(1)$ & $-C(13)$ & $-C(12)$ & $111.89(18)$ \\
\hline $\operatorname{Mn}(1)$ & $-C(14)$ & $-O(14)$ & $176.8(2)$ & $\operatorname{Mn}(1)$ & $-C(15)$ & $-O(15)$ & $176.4(2)$ \\
\hline Mn (1) & - C (16) & $-o(16)$ & $179.5(2)$ & Mn (2) & $-C(21)$ & $-C(22)$ & $70.21(11)$ \\
\hline $\operatorname{Mn}(2)$ & $-C(21)$ & $-C(26)$ & $70.12(12)$ & $C(22)$ & $-C(21)$ & $-C(26)$ & $116.93(19)$ \\
\hline Mn (2) & $-C(21)$ & $-C(28)$ & $127.86(14)$ & $C(22)$ & $-C(21)$ & $-C(28)$ & $122.16(19)$ \\
\hline$C(26)$ & $-C(21)$ & $-C(28)$ & $120.8(2)$ & $\operatorname{Mn}(2)$ & $-C(22)$ & $-C(21)$ & $71.82(12)$ \\
\hline $\operatorname{Mn}(2)$ & $-C(22)$ & $-C(23)$ & $72.29(13)$ & $C(21)$ & $-C(22)$ & - C (23) & $121.6(2)$ \\
\hline Mn (2) & $-C(23)$ & $-c(22)$ & $70.08(13)$ & $\operatorname{Mn}(2)$ & $-C(23)$ & $-C(24)$ & $75.03(15)$ \\
\hline$C(22)$ & $-C(23)$ & $-C(24)$ & $119.9(2)$ & Mn (2) & $-C(24)$ & $-O(27)$ & $127.6(2)$ \\
\hline $\operatorname{Mn}(2)$ & $-C(24)$ & $-O(271)$ & $130.2(2)$ & $O(27)$ & $-C(24)$ & $-O(271)$ & $33.33(18)$ \\
\hline $\operatorname{Mn}(2)$ & $-C(24)$ & $-C(23)$ & $68.23(13)$ & $O(27)$ & $-C(24)$ & $-C(23)$ & $135.8(3)$ \\
\hline
\end{tabular}


Table IV (continued)

$\begin{array}{lcllr}O(271)-C(24)-C(23) & 102.9(3) & M n(2)-C(24)-C(25) & 69.01(14) \\ O(27)-C(24)-C(25) & 104.4(3) & O(271)-C(24)-C(25) & 137.7(3) \\ C(23)-C(24)-C(25) & 119.3(2) & M n(2)-C(25)-C(24) & 74.91(15) \\ M n(2)-C(25)-C(26) & 69.38(13) & C(24)-C(25)-C(26) & 119.8(2) \\ M n(2)-C(26)-C(21) & 72.06(12) & M n(2)-C(26)-C(25) & 72.89(14) \\ C(21)-C(26)-C(25) & 122.0(2) & O(27)-C(27)-O(271) & 29.0(3) \\ O(27)-C(27)-C(271) & 37.6(3) & O(271)-C(27)-C(271) & 66.2(4) \\ C(21)-C(28)-C(29) & 121.24(19) & C(21)-C(28)-C(30) & 116.09(18) \\ C(29)-C(28)-C(30) & 122.65(19) & \mathrm{S}(2)-C(30)-C(28) & 120.22(15) \\ S(2)-C(30)-C(31) & 110.69(15) & C(28)-C(30)-C(31) & 128.98(18) \\ C(30)-C(31)-C(32) & 112.56(19) & C(31)-C(32)-C(33) & 113.1(2) \\ S(2)-C(33)-C(32) & 111.28(16) & M n(2)-C(34)-O(34) & 176.14(19) \\ M n(2)-C(35)-O(35) & 174.8(2) & M n(2)-C(36)-O(36) & 178.2(2) \\ O(27)-C(271)-O(271) & 31.4(3) & O(27)-C(271)-C(27) & 65.6(4) \\ O(271)-C(271)-C(27) & 34.5(3) & F(11)-B(1)-F(12) & 109.4(2) \\ F(11)-B(1)-F(13) & 111.2(2) & F(12)-B(1)-F(13) & 110.2(2) \\ F(11)-B(1)-F(14) & 109.3(2) & F(12)-B(1)-F(14) & 107.5(2) \\ F(13)-B(1)-F(14) & 109.1(2) & F(21)-B(2)-F(22) & 110.9(3) \\ F(21)-B(2)-F(23) & 107.3(2) & F(22)-B(2)-F(23) & 109.9(2) \\ F(21)-B(2)-F(24) & 109.3(2) & F(22)-B(2)-F(24) & 111.7(2) \\ F(23)-B(2)-F(24) & 107.5(2) & & & \end{array}$


Table S1 : Anisotropic thermal parameters for $\mathrm{C}_{16} \mathrm{H}_{12} \mathrm{BF}_{4} \mathrm{MnO}_{4} \mathrm{~S}$

\begin{tabular}{|c|c|c|c|c|c|c|}
\hline Atom & $\mathrm{u}(11)$ & $u(22)$ & $\mathrm{u}(33)$ & $\mathrm{u}(23)$ & $\mathrm{u}(13)$ & $\mathrm{u}(12)$ \\
\hline $\operatorname{Mn}(1)$ & $0.01643(13)$ & $0.01542(14)$ & $0.01695(14)$ & $0.00063(11)$ & $-0.00099(10)$ & $-0.00162(11)$ \\
\hline $\operatorname{Mn}(2)$ & $0.01454(14)$ & $0.01527(15)$ & $0.02396(15)$ & $-0.00123(11)$ & $-0.00133(11)$ & $-0.00200(11)$ \\
\hline$S(1)$ & $0.0212(2)$ & $0.0254(3)$ & $0.0331(3)$ & $-0.0017(2)$ & $0.0077(2)$ & $0.0011(2)$ \\
\hline$S(2)$ & $0.0138(2)$ & $0.0262(3)$ & $0.0262(2)$ & $-0.0005(2)$ & $0.00154(18)$ & $0.00114(19)$ \\
\hline$O(7)$ & $0.0173(7)$ & $0.0396(10)$ & $0.0328(9)$ & $-0.0013(7)$ & $0.0020(6)$ & $0.0062(7)$ \\
\hline$O(14)$ & $0.0300(8)$ & $0.0223(8)$ & $0.0475(10)$ & $-0.0035(7)$ & $0.0010(7)$ & $0.0030(7)$ \\
\hline$O(15)$ & $0.0401(10)$ & $0.0445(11)$ & $0.0195(7)$ & $-0.0032(7)$ & $-0.0023(7)$ & $-0.0008(8)$ \\
\hline$O(16)$ & $0.0304(9)$ & $0.0414(11)$ & $0.0524(12)$ & $0.0065(9)$ & $0.0007(8)$ & $-0.0165(8)$ \\
\hline$O(27)$ & $0.0128(15)$ & $0.0359(19)$ & $0.0287(18)$ & $0.0072(16)$ & $0.0010(13)$ & $0.0046(13)$ \\
\hline$O(34)$ & $0.0369(9)$ & $0.0203(8)$ & $0.0397(10)$ & $0.0010(7)$ & $0.0031(7)$ & $0.0055(7)$ \\
\hline$O(35)$ & $0.0441(11)$ & $0.0517(12)$ & $0.0234(9)$ & $0.0053(8)$ & $-0.0044(7)$ & $-0.0125(9)$ \\
\hline$O(36)$ & $0.0272(9)$ & $0.0495(13)$ & $0.0708(15)$ & $-0.0092(11)$ & $-0.0033(9)$ & $-0.0190(9)$ \\
\hline$O(271)$ & $0.0194(18)$ & $0.035(2)$ & $0.033(2)$ & $0.0042(16)$ & $0.0037(16)$ & $0.0031(14)$ \\
\hline$C(1)$ & $0.0198(9)$ & $0.0159(9)$ & $0.0187(9)$ & $-0.0012(7)$ & $0.0008(7)$ & $-0.0037(7)$ \\
\hline$C(2)$ & $0.0172(8)$ & $0.0177(9)$ & $0.0173(8)$ & $0.0011(7)$ & $-0.0009(7)$ & $-0.0017(7)$ \\
\hline$C(3)$ & $0.0178(9)$ & $0.0221(10)$ & $0.0184(9)$ & $-0.0010(7)$ & $-0.0005(7)$ & $-0.0022(7)$ \\
\hline$C(4)$ & $0.0171(9)$ & $0.0219(10)$ & $0.0254(10)$ & $-0.0046(8)$ & $0.0002(7)$ & $0.0020(8)$ \\
\hline$C(5)$ & $0.0238(10)$ & $0.0171(10)$ & $0.0287(11)$ & $0.0008(8)$ & $-0.0049(8)$ & $0.0031(8)$ \\
\hline$C(6)$ & $0.0263(10)$ & $0.0151(9)$ & $0.0219(10)$ & $0.0031(7)$ & $-0.0013(8)$ & $-0.0016(8)$ \\
\hline$C(7)$ & $0.0218(10)$ & $0.0465(15)$ & $0.0355(12)$ & $0.0009(11)$ & $0.0079(9)$ & $0.0009(10)$ \\
\hline$C(8)$ & $0.0205(9)$ & $0.0199(10)$ & $0.0233(9)$ & $0.0017(8)$ & $0.0036(7)$ & $-0.0027(8)$ \\
\hline$C(9)$ & $0.0293(11)$ & $0.0464(15)$ & $0.0249(11)$ & $0.0005(10)$ & $0.0058(9)$ & $-0.0036(11)$ \\
\hline$C(10)$ & $0.0157(9)$ & $0.0185(10)$ & $0.0273(10)$ & $-0.0018(8)$ & $0.0066(7)$ & $-0.0038(7)$ \\
\hline$C(11)$ & $0.0171(9)$ & $0.0247(11)$ & $0.0320(11)$ & $-0.0073(9)$ & $0.0029(8)$ & $-0.0058(8)$ \\
\hline$C(12)$ & $0.0240(11)$ & $0.0355(14)$ & $0.0387(13)$ & $-0.0130(11)$ & $-0.0010(9)$ & $-0.0052(10)$ \\
\hline$C(13)$ & $0.0210(10)$ & $0.0310(12)$ & $0.0434(13)$ & $-0.0030(10)$ & $0.0005(9)$ & $-0.0050(9)$ \\
\hline$C(14)$ & $0.0223(10)$ & $0.0206(10)$ & $0.0261(10)$ & $-0.0010(8)$ & $-0.0004(8)$ & $-0.0047(8)$ \\
\hline$C(15)$ & $0.0261(10)$ & $0.0234(11)$ & $0.0241(10)$ & $-0.0014(8)$ & $0.0013(8)$ & $-0.0018(8)$ \\
\hline$C(16)$ & $0.0237(10)$ & $0.0278(11)$ & $0.0263(11)$ & $-0.0011(9)$ & $-0.0014(8)$ & $-0.0027(9)$ \\
\hline$C(21)$ & $0.0160(9)$ & $0.0157(9)$ & $0.0288(10)$ & $-0.0052(8)$ & $-0.0006(7)$ & $-0.0025(7)$ \\
\hline$C(22)$ & $0.0170(9)$ & $0.0293(11)$ & $0.0277(10)$ & $-0.0127(9)$ & $0.0015(8)$ & $-0.0044(8)$ \\
\hline$C(23)$ & $0.0185(10)$ & $0.0393(14)$ & $0.0410(13)$ & $-0.0223(11)$ & $0.0109(9)$ & $-0.0078(9)$ \\
\hline$C(24)$ & $0.0141(10)$ & $0.0275(13)$ & $0.081(2)$ & $-0.0250(13)$ & $0.0036(11)$ & 0.0005 (9) \\
\hline$C(25)$ & $0.0204(11)$ & $0.0157(11)$ & $0.084(2)$ & $-0.0015(12)$ & $-0.0127(12)$ & $0.0026(9)$ \\
\hline$C(26)$ & $0.0215(10)$ & $0.0136(10)$ & $0.0518(15)$ & $0.0023(9)$ & $-0.0079(10)$ & $-0.0016(8)$ \\
\hline$C(27)$ & $0.020(2)$ & $0.060(4)$ & $0.035(3)$ & $0.014(3)$ & $0.010(2)$ & $0.005(2)$ \\
\hline$C(28)$ & $0.0142(8)$ & $0.0162(9)$ & $0.0258(9)$ & $0.0018(7)$ & $-0.0003(7)$ & $-0.0011(7)$ \\
\hline$C(29)$ & $0.0210(10)$ & $0.0403(14)$ & $0.0239(10)$ & $-0.0021(9)$ & $0.0003(8)$ & $-0.0015(9)$ \\
\hline$C(30)$ & $0.0135(8)$ & $0.0160(9)$ & $0.0247(9)$ & $0.0020(7)$ & $0.0013(7)$ & $-0.0022(7)$ \\
\hline$C(31)$ & $0.0172(9)$ & $0.0227(10)$ & $0.0285(11)$ & $-0.0035(8)$ & $0.0027(8)$ & $-0.0007(8)$ \\
\hline$C(32)$ & $0.0245(10)$ & $0.0278(12)$ & $0.0288(11)$ & $-0.0058(9)$ & $-0.0006(8)$ & $-0.0037(9)$ \\
\hline$C(33)$ & $0.0199(9)$ & $0.0278(11)$ & $0.0286(11)$ & $-0.0001(9)$ & $-0.0040(8)$ & $-0.0044(8)$ \\
\hline$C(34)$ & $0.0229(10)$ & $0.0175(10)$ & $0.0231(10)$ & $-0.0031(7)$ & $0.0031(8)$ & $-0.0032(8)$ \\
\hline$C(35)$ & $0.0264(10)$ & $0.0260(11)$ & $0.0284(11)$ & $0.0040(9)$ & $-0.0063(9)$ & $-0.0068(9)$ \\
\hline$C(36)$ & $0.0244(11)$ & $0.0278(12)$ & $0.0409(14)$ & $-0.0064(10)$ & $-0.0026(9)$ & $-0.0026(9)$ \\
\hline$C(271)$ & $0.019(2)$ & $0.041(3)$ & $0.051(3)$ & $0.011(2)$ & $0.002(2)$ & $0.010(2)$ \\
\hline$B(1)$ & $0.0274(12)$ & $0.0431(17)$ & $0.0269(12)$ & $0.0011(11)$ & $-0.0044(10)$ & $-0.0081(12)$ \\
\hline$B(2)$ & $0.0201(11)$ & $0.0403(16)$ & $0.0353(14)$ & $0.0074(12)$ & $-0.0015(10)$ & $-0.0032(11)$ \\
\hline$F(11)$ & $0.0515(11)$ & $0.0489(11)$ & $0.0663(12)$ & $0.0107(9)$ & $-0.0034(9)$ & $-0.0224(9)$ \\
\hline$F(12)$ & $0.0421(9)$ & $0.0584(11)$ & $0.0313(8)$ & $-0.0100(7)$ & $0.0018(6)$ & $-0.0110(8)$ \\
\hline F (13) & $0.0387(8)$ & $0.0825(14)$ & $0.0261(7)$ & $-0.0003(8)$ & $-0.0082(6)$ & $0.0054(9)$ \\
\hline$F(14)$ & $0.0423(9)$ & $0.0438(10)$ & $0.0568(11)$ & $0.0004(8)$ & $-0.0136(8)$ & $0.0018(8)$ \\
\hline$F(21)$ & $0.0431(10)$ & $0.0586(12)$ & $0.0717(13)$ & $-0.0007(10)$ & $-0.0083(9)$ & $-0.0197(9)$ \\
\hline$F(22)$ & $0.0418(10)$ & $0.125(2)$ & $0.0422(10)$ & $0.0313(12)$ & $0.0108(8)$ & $0.0216(12)$ \\
\hline F (23) & $0.0374(8)$ & $0.0442(9)$ & $0.0475(9)$ & $0.0000(8)$ & $-0.0143(7)$ & $0.0017(7)$ \\
\hline$F(24)$ & $0.0381(9)$ & $0.0543(12)$ & $0.0751(13)$ & $0.0272(10)$ & $0.0110(9)$ & $0.0129(8)$ \\
\hline
\end{tabular}


$\mathrm{C}_{16} \mathrm{H}_{12} \mathrm{BF}_{4} \mathrm{MnO}_{4} \mathrm{~S}$

Table S2 : Hydrogen atoms fractional atomic coordinates for

\begin{tabular}{|c|c|c|c|c|c|}
\hline Atom & $x / a$ & $\mathrm{y} / \mathrm{b}$ & $z / c$ & U (iso) & occ \\
\hline H (21) & 0.2071 & 0.8189 & 0.1716 & $0.0432(19)$ & \\
\hline H (31) & 0.3418 & 0.8249 & 0.1498 & $0.0432(19)$ & \\
\hline $\mathrm{H}(51)$ & 0.4065 & 0.4654 & 0.2507 & $0.0432(19)$ & \\
\hline H (61) & 0.2740 & 0.4847 & 0.2801 & $0.0432(19)$ & \\
\hline $\mathrm{H}(71)$ & 0.5357 & 0.6433 & 0.1275 & $0.0432(19)$ & \\
\hline $\mathrm{H}(72)$ & 0.4711 & 0.8107 & 0.1360 & $0.0432(19)$ & \\
\hline н (73) & 0.4443 & 0.6205 & 0.1091 & $0.0432(19)$ & \\
\hline H (91) & 0.1827 & 0.7031 & 0.3108 & $0.0432(19)$ & \\
\hline Н (92) & 0.0804 & 0.7015 & 0.3036 & $0.0432(19)$ & \\
\hline $\mathrm{H}(111)$ & 0.1232 & 0.4961 & 0.1583 & $0.0432(19)$ & \\
\hline $\mathrm{H}(121)$ & -0.0160 & 0.4904 & 0.1241 & $0.0432(19)$ & \\
\hline $\mathrm{H}(131)$ & -0.1169 & 0.6336 & 0.1731 & $0.0432(19)$ & \\
\hline $\mathrm{H}(221)$ & 0.1687 & 0.8298 & 0.4056 & $0.0432(19)$ & \\
\hline $\mathrm{H}(231)$ & 0.3105 & 0.8177 & 0.3973 & $0.0432(19)$ & \\
\hline $\mathrm{H}(251)$ & 0.3306 & 0.5158 & 0.5167 & $0.0432(19)$ & \\
\hline $\mathrm{H}(261)$ & 0.1888 & 0.5335 & 0.5257 & $0.0432(19)$ & \\
\hline $\mathrm{H}(271)$ & 0.5117 & 0.6487 & 0.4247 & $0.0432(19)$ & 0.5000 \\
\hline $\mathrm{H}(272)$ & 0.4456 & 0.8137 & 0.4165 & $0.0432(19)$ & 0.5000 \\
\hline $\mathrm{H}(273)$ & 0.4334 & 0.6077 & 0.3932 & $0.0432(19)$ & 0.5000 \\
\hline $\mathrm{H}(291)$ & 0.0840 & 0.7681 & 0.5398 & $0.0432(19)$ & \\
\hline $\mathrm{H}(292)$ & -0.0118 & 0.7541 & 0.5180 & $0.0432(19)$ & \\
\hline $\mathrm{H}(311)$ & 0.0952 & 0.4947 & 0.3909 & $0.0432(19)$ & \\
\hline $\mathrm{H}(321)$ & -0.0236 & 0.4592 & 0.3387 & $0.0432(19)$ & \\
\hline $\mathrm{H}(331)$ & -0.1442 & 0.6198 & 0.3669 & $0.0432(19)$ & \\
\hline H (2711) & 0.5176 & 0.5625 & 0.4519 & $0.0432(19)$ & 0.5000 \\
\hline H (2712) & 0.4628 & 0.6141 & 0.4952 & $0.0432(19)$ & 0.5000 \\
\hline H (2713) & 0.4453 & 0.4226 & 0.4665 & $0.0432(19)$ & 0.5000 \\
\hline
\end{tabular}

